\title{
Prevalence of autoimmune thyroiditis in patients with type 1 diabetes: a long-term follow-up study
}

\author{
Gum Bit Hwang, MD, \\ Jong Seo Yoon, MD, \\ Kyu Jung Park, MD, \\ Hae Sang Lee, MD, PhD, \\ Jin Soon Hwang, MD, PhD
}

Department of Pediatrics, Ajou University School of Medicine, Ajou University Hospital, Suwon, Korea

Received: 29 August, 2017

Revised: 29 September, 2017

Accepted: 24 October, 2017

Address for correspondence: Hae Sang Lee

Department of Pediatrics, Ajou University School of Medicine, 206 World cup-ro, Yeongtong-gu, Suwon 16499, Korea

Tel: +82-31-219-4454

Fax: +82-31-219-5169

E-mail: seaon98@naver.com

https://orcid.org/0000-0002-96844042
Purpose: Type 1 diabetes mellitus (DM) is associated with autoimmune diseases such as thyroiditis. Therefore, we aimed to investigate the prevalence of autoimmune thyroiditis in patients with type $1 \mathrm{DM}$.

Methods: A total of 102 patients who were diagnosed and followed up (mean age, 8.1 \pm 4.0 years) in Ajou University Hospital were enrolled in this study. All the patients were evaluated for beta cell autoimmunity, including insulin autoantibody, glutamic acid decarboxylase antibodies (GADA), and islet cell antibody. Moreover, autoantibodies to thyroid peroxidase and thyroglobulin were assessed at initial diagnosis and annually thereafter.

Results: The mean patient age (49 men and 53 women) was $19.2 \pm 4.8$ years. The prevalence of at least one thyroid antibody was $30.4 \%$. Patients with thyroid antibodies had a significantly higher frequency of GADA at the time of the diagnosis. Autoimmune thyroiditis was more prevalent in the older age group. GADA was a significant risk factor for development of thyroid autoantibodies after diagnosis of type 1 DM (odds ratio, 4.45; 95\% confidence interval, 1.399-14.153).

Conclusion: In patients with type $1 \mathrm{DM}$, the prevalence of autoimmune thyroiditis was higher than in the general population. Moreover, GADA positivity at diagnosis was associated with thyroid autoimmunity.

Keywords: Autoimmune thyroiditis, Type 1 diabetes, Thyroid autoantibody

\section{Introduction}

The immune-mediated destruction of pancreatic islet cells causes type 1 diabetes mellitus (DM). Other autoimmune diseases such as Addison disease, Hashimoto thyroiditis, Graves disease, and pernicious anemia are associated with type $1 \mathrm{DM}^{1{ }^{1}}$ In particular, autoimmune thyroiditis (AIT) is the most common disorder associated with type $1 \mathrm{DM}^{2)}$ AIT is characterized by $\mathrm{T}$ and B-lymphocyte infiltration of the thyroid gland and the presence of autoantibodies to thyroid peroxidase (TPO Ab) and thyroglobulin (TG Ab). ${ }^{3}$ AIT and type 1 DM have a common genetic background and similar pathogenesis; hence, they could occur in the same individual or family. The prevalence of thyroid autoantibodies in children with type 1 DM ranges from $3 \%$ to $50 \%$ in different countries and populations, which is markedly higher than in the general population (range, $1 \%$ to $4 \%$ ).

Thyroid autoantibodies can be detected at the initial diagnosis or can be detected over time, after diagnosis. ${ }^{5,6)}$ Age at diagnosis, pubertal status, and the female gender have been associated with thyroid autoantibody in children and adolescents with type $1 \mathrm{DM}^{7-10)}$ In addition, recent studies have reported that the presence of glutamic acid decarboxylase antibodies (GADA) and human leucocyte antigen class II genes may influence the development or progression of AIT. ${ }^{11,12)}$ However, a few studies have evaluated the prevalence and characteristics of AIT occurring with type $1 \mathrm{DM}$ in Korea. ${ }^{5,13,14)}$ Therefore, the aim of this study was to evaluate the prevalence of AIT and identify the factors associated with occurrence of thyroid 
autoantibodies in patients with type $1 \mathrm{DM}$.

\section{Materials and methods}

\section{Patients}

The study population included 102 patients with type $1 \mathrm{DM}$ who were treated in Ajou University Hospital from March 2003 to July 2017. The study design was reviewed and approved by the Institutional Review Board of Ajou University Hospital (AJIRB-MED-MDB-17-498). All the patients had been diagnosed with type $1 \mathrm{DM}$ according to the criteria of American Diabetes Association. ${ }^{15)}$ Patients with positive of thyroid autoantibodies (TPO Ab, TG Ab, or thyroidstimulating hormone [TSH] receptor-stimulating antibody) were considered to have AIT. Hypothyroidism was defined as an elevated TSH level $(>5 \mathrm{IU} / \mathrm{L})$ with or without decreased serum T3 or free T4 levels. The diagnosis of Graves disease was based on clinical manifestations and confirmed according to elevated serum free T4 and T3 levels, suppressed TSH levels and positive TSH receptor-stimulating antibodies. We collected clinical data including patients' height, weight, pubertal status, medical history, family history of diabetes or thyroid disease, and laboratory results from the clinical charts and electronic medical records. Body mass index was calculated as weight divided by height $\left(\mathrm{kg} / \mathrm{m}^{2}\right)$. Pubertal stage was determined by the Tanner and Marshal method. ${ }^{16)}$ Prepubertal stage was defined as the lack of breast development in girls and a testicular volume below $4 \mathrm{~mL}$ in boys.

\section{Laboratory measurements}

Laboratory analysis included serum free T4, T3, TSH, and thyroid autoantibodies (TPO Ab, TG Ab, and TSH receptorstimulating antibody) for all the patients at the initial diagnosis. Thyroid function tests and the autoantibody test were repeated at least once every year. The reference ranges were as follows: free T4, 0.64-1.72 ng/dL; T3, 76-190 ng/ dL; TSH, 0.15-5.00 IU/L; TPO Ab, 0-60 U/mL; TG Ab, 0-60 $\mathrm{U} / \mathrm{mL}$; and TSH receptor-stimulating antibody, 0-1.5 IU/L. Serum free T4, T3, and TSH concentrations were measured using radioimmunoassay methods (Auto RIA/SR300, Startec Biomedical AG, Birkenfeld, Germany). TPO Ab and TG Ab were measured by immunoradiometric assays (Packard Cobra II Gamma Counter, Perkin Elmer Life Sciences, Courtaboeuf, France). TSH receptor-stimulating antibody was measured by radio-receptor assay (TSH Rezak, Medipan Diagnostica, Germany). Insulin autoantibody (IAA), GADA, and islet cell antibody, as markers of beta cell autoimmunity, were measured once using radioimmunoassay (RSR, Cardiff, Wales, UK) at initial diagnosis. A positive response was defined as 0.9 units/ $\mathrm{mL}$ and higher GADA level or $7 \%$ and higher IAA. Serum HDL cholesterol, LDL cholesterol, and triglycerides were determined with esterase and oxidase enzyme reactions (TBA200-FR, Toshiba, Japan). Hemoglobin $\mathrm{A}_{1 \mathrm{c}}\left(\mathrm{Hb}_{\mathrm{lc}}\right)$ was measured using a turbidimetric inhibition immunoassay (COBAS Integra 800, Roche, Switzerland).

\section{Statistical analysis}

IBM SPSS Statistics ver. 21.0 (IBM Co., Armonk, NY, USA) was used to perform the statistical analysis. Data are expressed as means \pm standard deviation. The Student $t$-test was used to compare the clinical parameters of the patients in the groups with or without thyroid autoantibodies. Differences of distributions for categorical variables were tested with the chisquare test. To determine significant factors associated with thyroid autoantibody positivity in type $1 \mathrm{DM}$, a binary logistic regression test was performed for multivariate analysis with forward variable selection using the patient's age at the time of diagnosis, $\mathrm{HbA}_{1 \mathrm{c}}$, initial TSH levels, and the presence of GADA. Statistical significance was defined as $P<0.05$.

Table 1. Clinical and biochemical characteristics of patients with type 1 diabetes mellitus with or without thyroid autoantibodies

\begin{tabular}{lccc}
\hline Variable & Positive thyroid antibodies $(\mathrm{n}=31)$ & Negative thyroid antibodies $(\mathrm{n}=71)$ & $P$-value \\
\hline Current age $(\mathrm{yr})$ & $19.9 \pm 4.0$ & $18.9 \pm 0.9$ & 0.345 \\
Age at diagnosis of diabetes $(\mathrm{yr})$ & $10.3 \pm 3.4$ & $9.0 \pm 4.4$ & 0.113 \\
Sex, male:female & $16: 15$ & $33: 38$ & 0.671 \\
Duration of diabetes $(\mathrm{yr})$ & $8.6 \pm 4.1$ & $7.8 \pm 4.0$ & 0.353 \\
$\mathrm{HbA}_{1 \mathrm{c}}(\%)$ & $11.6 \pm 2.1$ & $12.4 \pm 2.2$ & 0.117 \\
TSH (IU/L) & $2.0 \pm 1.3$ & $2.2 \pm 1.5$ & 0.657 \\
Family history of diabetes & $4(12.9)$ & $12(16.9)$ & 0.771 \\
Beta cell autoantibody & & & \\
GAD & $25(80.6)$ & $43(60.6)$ & 0.036 \\
IAA & $8(25.8)$ & $24(33.8)$ & 0.487 \\
ICA & $3(9.7)$ & $3(4.2)$ & 0.361 \\
\hline
\end{tabular}

Values are presented as mean \pm standard deviation or number (\%).

$\mathrm{HbA}_{1 c}$ glycated hemoglobin; TSH, thyroid-stimulating hormone; GAD, glutamic acid decarboxylase; IAA, insulin autoantibody, ICA, islet cell antibody. 
Table 2. Binary logistic regression analysis of factors associated with thyroid autoantibody positivity in patients with type1 diabetes mellitus $\left(n=102, r^{2}=0.205, P<0.001\right)$

\begin{tabular}{lccccc}
\hline Variable & $\mathrm{B}$ & Standard error & $P$-value & Odds ratio & $95 \% \mathrm{Cl}$ \\
\hline Age at diagnosis & 0.140 & 0.065 & 0.032 & 1.15 & $(1.012-1.306)$ \\
Sex & 0.352 & 0.482 & 0.465 & 1.42 & $(0.553-3.655)$ \\
Duration of diabetes & 0.041 & 0.063 & 0.522 & 1.04 & $(0.920-1.179)$ \\
Initial TSH & 0.153 & 0.169 & 0.364 & 0.85 & $(0.605-0.954)$ \\
GADA & 1.493 & 0.590 & 0.011 & 4.45 & $(1.399-14.153)$ \\
\hline
\end{tabular}

Regression analysis contained the following independent variables entered in to the model: age at diagnosis, sex, duration of disease, initial TSH levels, and the presence of GADA.

$\mathrm{Cl}$, confidence interval; TSH, thyroid-stimulating hormone; GADA, glutamic acid decarboxylase antibodies.

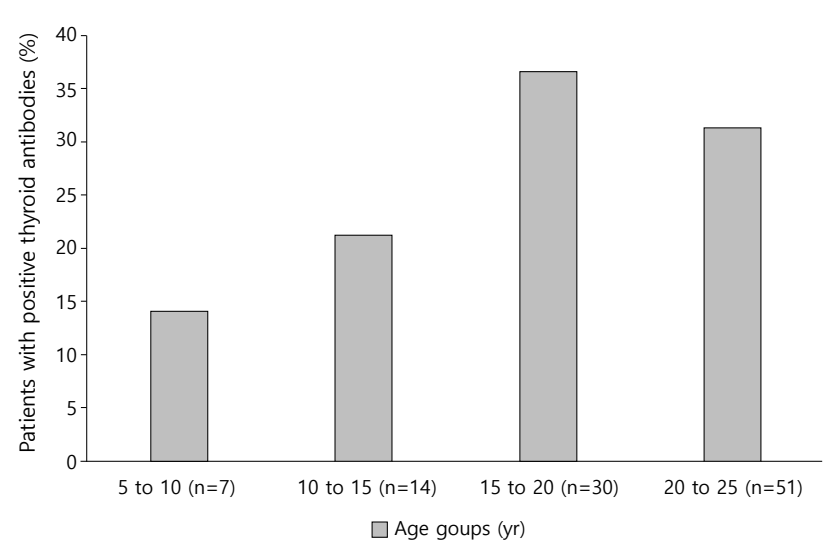

Fig. 1. Frequency of thyroid autoantibodies positivity in children with type 1 mellitus according to current age.

\section{Results}

The study population included 49 men (59.8\%) and 53 women $(40.2 \%)$ with a mean age of $19.2 \pm 4.8$ years (range, $8-25$ years). The initial mean $\mathrm{pH}$ and bicarbonate were $7.33 \pm 0.13$ and $18.6 \pm 0.6 \mathrm{mEq} / \mathrm{L}$, respectively. Thirty-five patients (34.3\%) were diagnosed with diabetic ketoacidosis (DKA) at the initial presentation. The mean duration of follow-up was $8.1 \pm 4.0$ years (range, $0.1-19.0$ years).

The rate at which at least one of the thyroid autoantibodies tested positive (TPO Ab or TG $\mathrm{Ab}$ ) was $30.4 \%(\mathrm{n}=31)$. Fifteen of all the patients (14.7\%) had TPO Ab and $23(22.5 \%)$ had TG Ab. Ten patients (9.8\%) had both TPO Ab and TG Ab. Three patients were diagnosed with Graves disease, so they received an antithyroid drug. Thirteen of the total patients (12.7\%) had thyroid autoantibodies at the initial diagnosis and 18 patients were detected with thyroid autoantibodies at a mean of $2.7 \pm 2.8$ years after diagnosis (mean age, $14.5 \pm 3.5$ years). All patients who were diagnosed with Hashimoto thyroiditis showed an euthyroid state and did not need to receive any thyroid drugs for the follow-up duration. The prevalence of positive GADA was significantly higher in patients with thyroid autoantibody (Table 1). None of the subjects had a family history of thyroid disease. There was also no difference between the 2 groups in their family history of diabetes. The occurrence of DKA at the time of diagnosis and serum cholesterol levels were not significantly different between the patients with or without thyroid autoantibodies. Moreover, the older age group showed a higher ratio of positivity for thyroid autoantibodies compared to the younger age group, although not statistically significant (Fig. 1).

In a binary logistic regression analysis with age at diagnosis, sex, duration of disease, initial TSH levels, and the presence of GADA as independent variables; and positivity for thyroid autoantibodies as the dependent variable, the initial presence of GADA was a significant predictor of the presence of thyroid autoantibodies (Table 2). Additionally, increasing age was correlated with the development of AIT.

\section{Discussion}

In this study, the prevalence of AIT observed in patients with type $1 \mathrm{DM}$ was $30.4 \%$, and the odds ratio of having thyroid autoantibodies was higher in the older age group and in patients with GADA.

Previous studies that evaluated the prevalence of thyroid autoantibodies in patients with type 1 DM reported these patients to have a high frequency of AIT, but these studies also demonstrated a very wide range of prevalence. ${ }^{2)}$ Lee et al. ${ }^{13)}$ evaluated the prevalence of AIT in 139 patients with type 1 DM and $38.8 \%$ of these patients had at least one of the thyroid autoantibodies. In another study by Kang et al., ${ }^{14)}$ the prevalence of AIT was $28.8 \%$, similar to our results. A recent study reported that thyroid autoantibodies positivity was detected in $26 \%$ of type 1 DM patients at the initial diagnosis. ${ }^{9)}$ In the general population, the rate of AIT ranges from $0.1 \%$ to $2 \%$, but its prevalence in children and adolescents with type $1 \mathrm{DM}$ is higher than in the nondiabetic general population of the same age group. ${ }^{6.17)}$ Moreover, children with type 1 DM have a 24-fold risk of developing thyroid disease in a nationwide cohort study. ${ }^{18)}$ Hence, the American Diabetic Association and International Society for Pediatric and Adolescent Diabetes recommend the screening of thyroid function and autoantibodies at the initial diagnosis, and the regular screening of thyroid function even in asymptomatic patients. ${ }^{19)}$

Many studies have demonstrated that several factors such as age, the female sex, duration of disease, and the presence of beta cell autoimmunity are associated with AIT in type 1 DM patients. ${ }^{9,10,13,20-22)}$ In our study, GADA positivity at the onset of type 1 DM was a predictor of AIT development. In 
another study by Jin et al., ${ }^{11)}$ a high GADA titer was a significant predictor of AIT development in type 1 DM patients. The exact mechanism underlying the effect of GADA on thyroid autoimmunity is unclear. GADA is one of the pancreatic islet autoantibodies and can also be found in the brain and thyroid gland. ${ }^{23)}$ Several studies have reported an elevated GADA titer in patients with Hashimoto thyroiditis and Graves disease compared to normal controls. ${ }^{24,25)}$ Moreover, type 1 DM and AIT have a common genetic background of shared susceptibility. ${ }^{24)}$ These results suggest that GADA positivity in type $1 \mathrm{DM}$ patients is associated with the development of AIT. Other islet autoantibodies were also associated with thyroid autoantibodies. ${ }^{9,26)}$ In Korean study by Jung et al., ${ }^{9)}$ the IAA positivity at initial diagnosis was correlated with positivity of thyroid autoantibodies. Jonsdottir et al. ${ }^{7)}$ reported that zinc transporter 8 antibody as well as GADA positivity at initial diagnosis increased the risk of thyroid dysfunction.

In addition, thyroid autoantibodies were more prevalent in the older age group in our study. These findings are consistent with previous reports. ${ }^{1,2)}$ Kordonouri et al. ${ }^{22)}$ reported that the prevalence of patients with thyroid autoantibodies in type 1 DM was significantly increased with increasing patient age. In a study by Riquetto et al.," patients in the older age group had a tendency for a higher incidence of AIT. Also, there have been many studies in which AIT occurred more frequently in female compared to male type $1 \mathrm{DM}$ patients. ${ }^{22,27)}$ In a recent review, androgens had a protective effect against the progression of autoimmunity, while estradiol seemed to accelerate the progression of this autoimmune disease through the T-lymphocytes pathway. ${ }^{28)}$ However, there was no statistical difference between the female and male patients with type 1 DM in our study. These differences may be due to differences in ethnic backgrounds and study sample size. In addition, the development of AIT increased with increasing disease duration in children and adolescents with type $1 \mathrm{DM}^{27,29)}$

This study had limitations stemming from its small sample size and retrospective study design. Prospective longitudinal studies with more patients would be needed to characterize the association between thyroid autoimmunity and type $1 \mathrm{DM}$.

In conclusion, our results showed that AIT occurred more frequently in patients with type $1 \mathrm{DM}$. In particular, the presence of GADA was a significant risk factor for the development of thyroid autoimmunity. Therefore, regular screening of thyroid function and thyroid autoantibodies may be considered in all type 1 DM patients.

\section{Conflict of interest}

No potential conflict of interest relevant to this article was reported.

\section{References}

1. Barker JM. Clinical review: type 1 diabetes-associated autoimmunity: natural history, genetic associations, and screening. J Clin Endocrinol Metab 2006;91:1210-7.

2. Shun CB, Donaghue KC, Phelan H, Twigg SM, Craig ME. Thyroid autoimmunity in type 1 diabetes: systematic review and meta-analysis. Diabet Med 2014;31:126-35.

3. Dayan CM, Daniels GH. Chronic autoimmune thyroiditis. N Engl J Med 1996;335:99-107.

4. Kahaly GJ, Hansen MP. Type 1 diabetes associated autoimmunity. Autoimmun Rev 2016;15:644-8.

5. Oh KY, Kim YH, Yang EM, Kim CJ. Frequency of diabetes and thyroid autoantibodies in patients with type 1 diabetes and their siblings. Chonnam Med J 2016;52:136-40.

6. Kordonouri O, Hartmann R, Deiss D, Wilms M, GrütersKieslich A. Natural course of autoimmune thyroiditis in type 1 diabetes: association with gender, age, diabetes duration, and puberty. Arch Dis Child 2005;90:411-4.

7. Jonsdottir B, Larsson C, Carlsson A, Forsander G, Ivarsson SA, Lernmark A, et al. Thyroid and islet autoantibodies predict autoimmune thyroid disease at type 1 diabetes diagnosis. J Clin Endocrinol Metab 2017;102:1277-85.

8. Riquetto AD, de Noronha RM, Matsuo EM, Ishida EJ, Vaidergorn RE, Soares Filho MD, et al. Thyroid function and autoimmunity in children and adolescents with Type 1 Diabetes Mellitus. Diabetes Res Clin Pract 2015;110:e9-11.

9. Jung ES, Han DK, Yang EM, Kim MS, Lee DY, Kim CJ. Thyroid autoimmunity in children and adolescents with newly diagnosed type 1 diabetes mellitus. Ann Pediatr Endocrinol Metab 2014;19:76-9.

10. Reghina AD, Albu A, Petre N, Mihu M, Florea S, Fica S. Thyroid autoimmunity in 72 children with type 1 diabetes mellitus: relationship with pancreatic autoimmunity and child growth. J Pediatr Endocrinol Metab 2012;25:723-6.

11. Jin P, Huang G, Lin J, Yang L, Xiang B, Zhou W, et al. High titre of antiglutamic acid decarboxylase autoantibody is a strong predictor of the development of thyroid autoimmunity in patients with type 1 diabetes and latent autoimmune diabetes in adults. Clin Endocrinol (Oxf) 2011;74:587-92.

12. Im EY, Shin CH, Yang SW. Polymorphisms of HLA class II predispose children and adolescents with type 1 diabetes mellitus to autoimmune thyroid disease. Autoimmunity 2003;36:177-81.

13. Lee SM, Chung HR, Hong SY, Shin CH, Yang SW. Clinical characteristics of autoimmune thyroid disease developed in patients with type 1 diabetes mellitus. Korean J Pediatr 2005;48:292-7.

14. Kang SY, Shin CH, Yang SW, Park MH, Yu J. Human leukocyte antigen (HLA) genotypes and thyroid autoimmunity in Korean patients with type 1 diabetes. Korean J Pediatr 2005 Jun;48:624-33.

15. American Diabetes Association. 2. Classification and diagnosis of diabetes. Diabetes Care 2017;40(Suppl 1):S1124.

16. Marshall WA, Tanner JM. Variations in pattern of pubertal changes in girls. Arch Dis Child 1969;44:291-303.

17. Hunter I, Greene SA, MacDonald TM, Morris AD. 
Prevalence and aetiology of hypothyroidism in the young. Arch Dis Child. 2000;83:207-10.

18. Spaans E, Schroor E, Groenier K, Bilo H, Kleefstra N, Brand P. Thyroid disease and type 1 diabetes in dutch children: a nationwide study (Young Dudes-3). J Pediatr 2017;187:18993.e1.

19. American Diabetes Association. 12. Children and adolescents. Diabetes Care 2017;40(Suppl 1):S105-13.

20. Libman IM, Sun K, Foley TP, Becker DJ. Thyroid autoimmunity in children with features of both type 1 and type 2 diabetes. Pediatr Diabetes 2008;9(4 Pt 1):266-71.

21. Mantovani RM, Mantovani LM, Dias VM. Thyroid autoimmunity in children and adolescents with type 1 diabetes mellitus: prevalence and risk factors. J Pediatr Endocrinol Metab 2007;20:669-75.

22. Kordonouri O, Klinghammer A, Lang EB, Grüters-Kieslich A, Grabert M, Holl RW. Thyroid autoimmunity in children and adolescents with type 1 diabetes: a multicenter survey. Diabetes Care 2002;25:1346-50.

23. Tsirogianni A, Pipi E, Soufleros K. Specificity of islet cell autoantibodies and coexistence with other organ specific autoantibodies in type 1 diabetes mellitus. Autoimmun Rev 2009;8:687-91.
24. Moriguchi M, Noso S, Kawabata Y, Yamauchi T, Harada T, Komaki K, et al. Clinical and genetic characteristics of patients with autoimmune thyroid disease with anti-islet autoimmunity. Metabolism 2011;60:761-6.

25. Kawasaki E, Takino H, Yano M, Uotani S, Matsumoto K, Takao Y, et al. Autoantibodies to glutamic acid decarboxylase in patients with IDDM and autoimmune thyroid disease. Diabetes 1994;43:80-6.

26. Jonsdottir B, Andersson C, Carlsson A, Delli A, Forsander G, Ludvigsson J, et al. Thyroid autoimmunity in relation to islet autoantibodies and HLA-DQ genotype in newly diagnosed type 1 diabetes in children and adolescents. Diabetologia 2013;56:1735-42.

27. Karavanaki K, Kakleas K, Paschali E, Kefalas N, Konstantopoulos I, Petrou V, et al. Screening for associated autoimmunity in children and adolescents with type 1 diabetes mellitus (T1DM). Horm Res 2009;71:201-6.

28. Ngo ST, Steyn FJ, McCombe PA. Gender differences in autoimmune disease. Front Neuroendocrinol 2014;35:34769.

29. Lorini R, d'Annunzio G, Vitali L, Scaramuzza A. IDDM and autoimmune thyroid disease in the pediatric age group. J Pediatr Endocrinol Metab 1996;9 Suppl 1:89-94. 